\title{
THE STABILITY AND RESPONSE OF A FLEXIBLE ROD IN A QUICK RETURN MECHANISM
}

\author{
D. G. BeAle \\ Auburn University, Auburn, Alabama 36849, U.S.A. \\ AND \\ R. A. SCOTt \\ Department of Mechanical Engineering and Applied Mechanics, The University of Michigan, \\ Ann Arbor, Michigan 48109, U.S.A.
}

(Received 12 June 1989, and in final form 14 November 1989)

\begin{abstract}
A quick return mechanism is analyzed for deflection and stability when the rod is considered an Euler-Bernoulli beam. The crank is assumed rigid and to be rotating at a constant angular velocity. The equations of motion and natural boundary conditions are obtained using Hamilton's principle. Spatial dependence is suppressed using Galerkin's method with time dependent pinned-pinned overhanging beam modes. Using a small crank length approximation, zones of parametric resonance are found using Hsu's method. The accuracy of these is verified using a monodromy matrix technique. The technique is also used to explore the possibility of resonances not covered by Hsu's (first order) method. A particular solution instability is found to exist using Hsu's method and is verified by direct numerical integration. For a somewhat flexible configuration, all instabilities were found to lie outside the range of normal operating speeds. For stiffer configurations likely to be found in practice, this conclusion can be asserted even stronger, at least for small cranks.
\end{abstract}

\section{INTRODUCTION}

As operating speeds increase and weight decreases, flexibility of links in various mechanisms has to be addressed. For relatively simple mechanisms, such as four bars and slider cranks, a considerable body of literature exists addressing response and stability; for examples, see references [1-7].

The mechanism analyzed here is a quick return one which has numerous industrial applications. For example, Ham, Crane and Rogers [8] described its use as a crank shaper. Sandor and Erdman [9] gave an example of its use in connection with a flow metering pump. Dwivedi [10] employed this mechanism for constructing a high velocity impacting press.

The quick return mechanism considered here has a flexible rod and a rigid crank as shown in Figure 1. A novel feature of the current work is that the flexible rod is supported in a translating joint that moves relative to the rod. The literature in the area of moving joints is sparse. For examples, see the papers by Buffington and Kane [11] on a magnetic tape problem, by Gürgöze [12] on a sewing machine mechanism, by Pan [13] on a prismatic joint robot arm, by Bahgat and Willmert [14] and by Song and Haug [15] on the quick return mechanism using finite element methods.

Many commercial codes for analyzing mechanisms, such as DADS [16], do not employ finite element methods, assumed mode techniques being more popular. The latter is the 


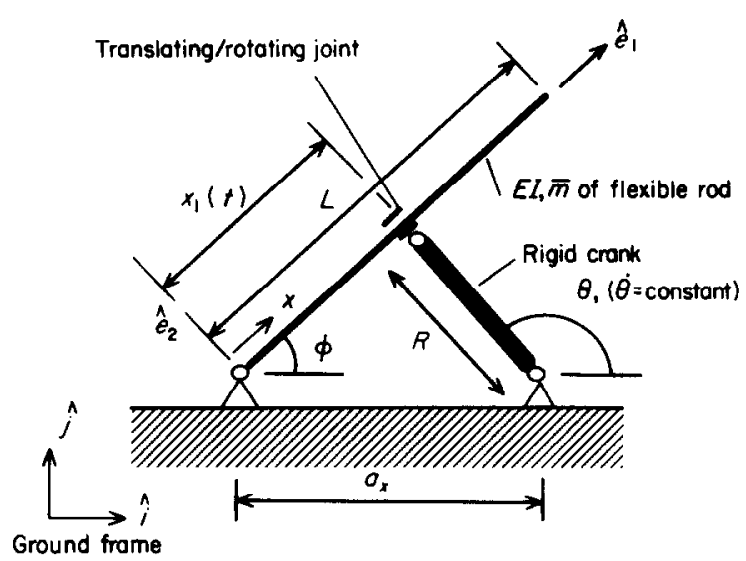

(a)

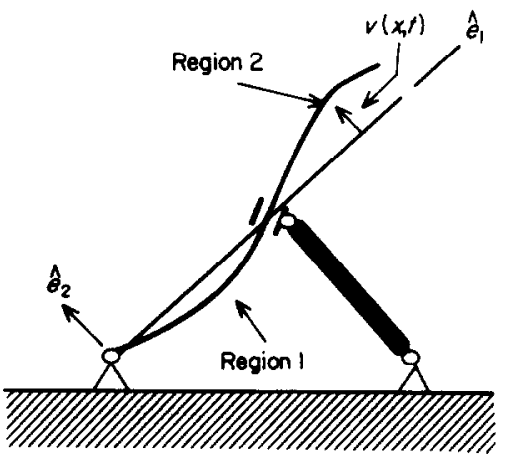

(b)

Figure 1. Rigid crank, flexible rod, quick return mechanism: (a) undeformed initial state; (b) deformed state.

approach adopted here. Galerkin's method is the technique used, with trial functions that are time dependent. This can lead to very considerable difficulties when the crank length is large (an approximate method for this problem will be presented in a later paper). Considerable simplification occurs when the length is small and that is the case pursued here. The authors believe the small crank case has merits in its right. In addition, it provides a benchmark solution against which other solutions (such as the approximate one to be presented in the later paper on the large crank) can be assessed.

Response and stability are treated. Response is obtained by numerical integration of the differential equations. Zones of parametric resonance of homogeneous equation instabilities are delineated by Hsu's perturbation method [17], and verified by the monodromy matrix technique (see reference [18]). A particular equation instability is also discovered to occur. Such instabilities have received little attention in the literature, as far as developing analytical techniques required to investigate them. Jandratis and Lowen [3], for example, investigated this instability approximately by decoupling the equations of motion by setting off-diagonal terms to zero. Herein no such simplifications are made.

\section{EQUATIONS OF MOTION}

The mechanism studied consists of a rotating crank driving a rod by means of a translating rotating joint (see Figure 1). Quick return mechanisms frequently have a link 
and a mass at the tip of the rod, but in some instances they are small and they are ignored here. The following modelling assumptions are made: (1) the crank is assumed rigid; (2) the rod is taken to be flexible and to be described by Euler-Bernoulli beam theory; (3) the translating rotating joint is treated as a "knife-edge"; $(4)$ the motion of the crank is taken to be prescribed as a constant angular velocity $\dot{\theta} ;(5)$ gravitational forces and joint friction are neglected.

The position vector from the left pin in the ground to any material point in the flexible rod is given by

$$
\bar{r}=x \hat{e}_{1}+v \hat{e}_{2} .
$$

The frame $\hat{e}_{1}, \hat{e}_{2}$ moves with angular velocity $\dot{\phi}$, where

$$
x_{1} \dot{\phi}=R \dot{\theta} \cos (\theta-\phi),
$$

where $x_{1}$ denotes the position of the moving joint along $\hat{e}_{1}$ and $R$ is the crank length.

The kinetic energy $T$ can be shown to be

$$
T=\frac{\bar{m}}{2}\left(\int_{0}^{x_{1}(t)}+\int_{x_{1}(t)}^{L}\right)\left(\left(\frac{\partial v}{\partial t}\right)^{2}+2 x \frac{\partial v}{\partial t} \dot{\phi}+x^{2} \dot{\phi}^{2}+v^{2} \dot{\phi}^{2}\right) \mathrm{d} x+\frac{1}{8} M_{c} R \theta^{2}+\frac{1}{2} J_{c} \dot{\theta}^{2},
$$

where $J_{c}$ is the mass moment of inertia of the crank about its center of mass, $M_{c}$ is the mass of the crank, and $\bar{m}$ is the mass per unit length of the rod, which is of length $L$. The potential energy is given by

$$
V=\frac{E I}{2}\left(\int_{0}^{x_{1}}+\int_{x_{1}}^{L}\right)\left(\frac{\partial^{2} v}{\partial x^{2}}\right)^{2} \mathrm{~d} x
$$

where $E I$ is the flexural rigidity of the rod. Hamilton's Principle involving a moving boundary has been treated by Forsyth [19]. By following that procedure, the nondimensional equations of motion can be shown to be (see reference [20] for details):

$$
\begin{gathered}
\frac{\lambda^{2}}{\dot{\theta}^{2}} \frac{\partial^{4} w}{\partial \eta^{4}}+\frac{\partial^{2} w}{\partial \theta^{2}}+\frac{1}{\left(1+\varepsilon^{2}+2 \varepsilon \cos \theta\right)^{2}}\left[\eta \varepsilon\left(\varepsilon^{2}-1\right) \sin \theta-w \varepsilon^{2}(\cos \theta+\varepsilon)^{2}\right]=0, \\
0<\eta<\bar{l}, \quad \bar{l}<\eta<1,
\end{gathered}
$$

where

$$
\theta=\dot{\theta} t, \quad w(\eta, \theta)=\frac{v(x, t)}{L}, \quad \varepsilon=\frac{R}{a_{x}}, \quad \eta=\frac{x}{L}, \quad \bar{l}=\frac{x_{1}}{L}, \quad \lambda^{2}=\frac{E I}{\bar{m} L^{4}},
$$

in which $a_{x}$ is the base length. The non-dimensional essential boundary conditions are

$$
w(\bar{l}, \theta)=0, \quad \frac{\partial w}{\partial \eta}\left(\bar{l}^{+}, \theta\right)=\frac{\partial w}{\partial \eta}\left(\bar{l}^{-}, \theta\right), \quad w(0, \theta)=0
$$

and the non-dimensional natural boundary conditions are

$$
\frac{\partial^{2} w}{\partial \eta^{2}}\left(\bar{l}^{+}, \theta\right)=\frac{\partial^{2} w}{\partial \eta^{2}}\left(\bar{l}^{-}, \theta\right), \quad \frac{\partial^{2} w}{\partial \eta^{2}}(0, \theta)=0, \quad \frac{\partial^{2} w}{\partial \eta^{2}}(1, \theta)=0, \quad \frac{\partial^{3} w}{\partial \eta^{3}}(1, \theta)=0 .
$$

\section{GALERKIN'S METHOD}

To solve equation (5) approximate methods must be employed. Here, following Gürgöze [12], Galerkin's method is used, in a somewhat non-standard fashion in that moving boundaries are involved. The following trial functions are used:

$$
w_{T_{1}}(\eta, \theta)=\sum_{n=1}^{N} f_{n}(\theta) e_{n 1}(\eta, \theta), \quad 0<\eta<\bar{l} ; \quad w_{T_{2}}(\eta, \theta)=\sum_{n=1}^{N} f_{n}(\theta) e_{n 2}(\eta, \theta), \quad T<\eta<1 .
$$


Here $e_{n 1}, e_{n 2}$ are the time dependent mode shapes of a pinned-pinned beam with an overhang for regions 1 and 2, respectively (see Figure 1). Expressions for them are given in reference [20].

In operator notation, equation (5) can be written as

$$
D(w)=g \text {. }
$$

Galerkin's method then leads to, for $k=1, \ldots, N$,

$$
\int_{0}^{T}\left(D\left(\sum_{n=1}^{N} f_{n} e_{n 1}\right)-g\right) e_{k 1} \mathrm{~d} \eta+\int_{i}^{1}\left(D\left(\sum_{n=1}^{N} f_{n} e_{n 2}\right)-g\right) e_{k 2} \mathrm{~d} \eta=0
$$

which leads to the ordinary differential equations

$$
\begin{gathered}
\Delta_{k k} \frac{\mathrm{d}^{2} f_{k}}{\mathrm{~d} \theta^{2}}+\sum_{n=1}^{N} \bar{a}_{k n} \frac{\mathrm{d} f_{n}}{\mathrm{~d} \theta}+f_{k}\left(\frac{\omega_{k}^{2}}{\dot{\theta}^{2}} \Delta_{k k}-\frac{\varepsilon^{2}(\cos \theta+\varepsilon)^{2}}{\left(1+2 \varepsilon \cos \theta+\varepsilon^{2}\right)^{2}} \Delta_{k k}\right)+\sum_{n=1}^{N} \bar{b}_{k n} f_{n} \\
=\frac{-\varepsilon \sin \theta\left(\varepsilon^{2}-1\right)}{\left(1+2 \varepsilon \cos \theta+\varepsilon^{2}\right)^{2}} \bar{g}_{k},
\end{gathered}
$$

where

$$
\begin{gathered}
\Delta_{k k}=\int_{0}^{\bar{T}} e_{k 1} e_{k 1} \mathrm{~d} \eta+\int_{T}^{1} e_{k 2} e_{k 2} \mathrm{~d} \eta, \quad \bar{a}_{k n}=2\left(\int_{0}^{\bar{T}} e_{k 1} \frac{\partial e_{n 1}}{\partial \theta} \mathrm{d} \eta+\int_{\bar{I}}^{1} e_{k 2} \frac{\partial e_{n 2}}{\partial \theta} \mathrm{d} \eta\right), \\
\bar{b}_{k n}=\int_{0}^{\bar{l}} e_{k 1} \frac{\partial^{2} e_{n 1}}{\partial \theta^{2}} \mathrm{~d} \eta+\int_{T}^{1} e_{k 2} \frac{\partial^{2} e_{n 2}}{\partial \theta^{2}} \mathrm{~d} \eta, \quad \bar{g}_{k}=\int_{0}^{T} \eta e_{k 1} \mathrm{~d} \eta+\int_{T}^{1} \eta e_{k 2} \mathrm{~d} \eta .
\end{gathered}
$$

$e_{k 1}$ and $e_{k 2}$ are very complicated and obtaining analytical expressions for the above coefficients is well-nigh intractable. Numerical integration is feasible, but turned out to be very CPU intensive due to the time dependent mode shapes. An approximate method, and an alternative mode method (which does not readily lend itself to stability studies), for the large crank will be presented in a later paper. Here, as in reference [12], attention is restricted to a small crank $(\varepsilon \ll 1)$ for which considerable simplification is achieved by use of Taylor series expansions.

\section{SMALL CRANK APPROXIMATION}

From geometry, it follows that, where $\bar{a}=a_{x} / L$,

$$
\bar{l}=\bar{a}\left(1+2 \varepsilon \cos \varepsilon+\varepsilon^{2}\right)^{1 / 2},
$$

which reduces to, for small $\varepsilon$, by using the binomial expansion,

$$
\bar{l}(\varepsilon, \theta)=\bar{a}(1+\varepsilon \cos \theta) \text {. }
$$

By using this expression and Taylor series expansions it can be shown, after substantial algebra (details can be found in reference [20]) that equations (12) become $\frac{\mathrm{d}^{2} f_{k}}{\mathrm{~d} \theta^{2}}+\frac{\omega_{k}^{2}}{\dot{\theta}^{2}} f_{k}+\varepsilon\left(\cos \theta \sum_{n=1}^{N} d_{k n}^{(1)} f_{n}+\sin \theta \sum_{n=1}^{N} g_{k n}^{(1)} \frac{\mathrm{d} f_{n}}{\mathrm{~d} \theta}\right)=\varepsilon \frac{\bar{g}_{k 1}}{\Delta_{k k 0}} \sin \theta, \quad k$

$$
=1, \ldots, N \text {, }
$$

where $d_{k n}^{(1)}, g_{k n}^{(1)}, \bar{g}_{k 1}$, and $\Delta_{k k 0}$ are complicated parameters (again details can be found in reference [20]) that depend, in general, on material properties, system geometry, and crank speed. $\omega_{k}$ is the $k$ th pinned-pinned overhanging beam natural frequency when the crank has zero length. Equations (19) are a non-homogeneous set of coupled linear ordinary differential equations with periodic coefficients. The response can be obtained using numerical integration. Instabilities arise through either the homogeneous solution or the non-homogeneous solution and these will be addressed separately. 


\section{RESPONSE}

Equations (19) were solved by using an Adams Moulton integrator on an APOLLO DN3000 computer to obtain the forced response. The properties used in reference [12] were used namely,

$$
\begin{gathered}
L=1 \mathrm{~m}, \quad a_{x}=0.59997 \mathrm{~m}, \quad E=0.7 \mathrm{E} 11 \mathrm{~N} / \mathrm{m}^{2}, \\
I=0.5208 \mathrm{E}-6 \mathrm{~m}^{4}, \quad \bar{m}=7.15 \mathrm{~kg} / \mathrm{m} .
\end{gathered}
$$

The tip deflections for a crank speed of $100 \mathrm{rad} / \mathrm{s}$ and $\varepsilon=0.01$, when using one, two and three modes, is plotted on Figure 2 as a function of the crank angle. It is seen that a one mode approximation captures most of the deflection, and the two and three mode solutions overlay each other. On the basis of this result, retention of just two modes was felt to be sufficiently accurate for the purpose of stability investigations. Note that the high frequency oscillations are due to excitation of, primarily, the first mode of vibration.

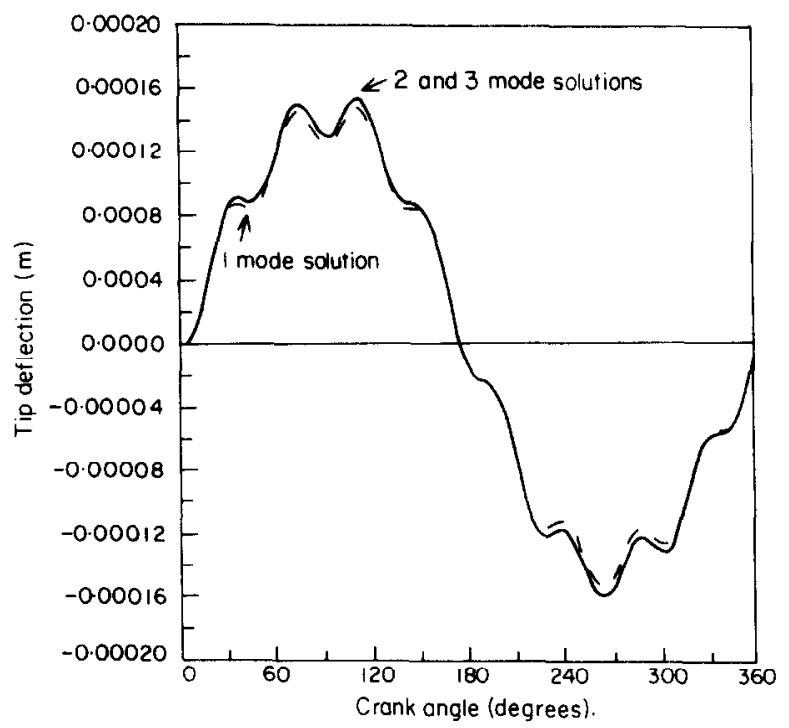

Figure 2. Small crank tip deflection; 1, 2 and 3 mode solutions.

\section{HOMOGENEOUS EQUATION INSTABILITIES}

Consider equations (19) with $\overline{\mathrm{g}}_{k_{1}}$ set equal to zero. The first derivative terms lead to complications in the stability analysis in that classical approaches involving Hill's infinite determinant (see reference [21]) fail to capture combination resonance zones. Hsu's method [17] does not have this drawback and it is the technique employed here. The method gives the following stability results.

\subsection{CASE A: RESONANCE AT CRANK SPEEDS NEAR TWICE THE FIRST}

\section{NATURAL FREQUENCY}

The stability-instability boundaries in the $\dot{\theta}, \varepsilon$ plane emanate from $\dot{\theta}$ near $2 \omega_{1}$. The stability-instability boundaries are defined by

$$
2 \frac{\omega_{1}}{\dot{\theta}} \pm \frac{\varepsilon\left(d_{11}^{(1)} \dot{\theta}-g_{11}^{(1)} \omega_{1}\right)}{2 \omega_{1}}=1
$$


The zone of instability is defined by:

$$
2 \frac{\omega_{1}}{\dot{\theta}}-\frac{\varepsilon\left(d_{11}^{(1)} \dot{\theta}-g_{11}^{(1)} \omega_{1}\right)}{2 \omega_{1}}<1<2 \frac{\omega_{1}}{\dot{\theta}}+\frac{\varepsilon\left(d_{11}^{(1)} \dot{\theta}-g_{11}^{(1)} \omega_{1}\right)}{2 \omega_{1}},
$$

and neutrally stable otherwise.

\subsection{CASE B: COMBINATION RESONANCE OF THE SUM TYPE}

The stability boundaries in the $\dot{\theta}, \varepsilon$ plane emanate from $\dot{\theta}$ near $\omega_{1}+\omega_{2}$. The stabilityinstability boundaries are defined by

$$
\frac{\omega_{1}}{\dot{\theta}}+\frac{\omega_{2}}{\dot{\theta}} \pm \frac{\varepsilon \dot{\theta}}{2 \sqrt{\omega_{1} \omega_{2}}} \sqrt{\left(d_{12}^{(1)}-g_{12}^{(1)} \frac{\omega_{2}}{\dot{\theta}}\right)\left(d_{21}^{(1)}+g_{21}^{(1)} \frac{\omega_{1}}{\dot{\theta}}\right)}=1
$$

The region between the boundaries is unstable if

$$
\begin{aligned}
\frac{\omega_{1}}{\dot{\theta}}+ & \frac{\omega_{2}}{\dot{\theta}}-\frac{\varepsilon \dot{\theta}}{2 \sqrt{\omega_{1} \omega_{2}}} \sqrt{\left(d_{12}^{(1)}-g_{12}^{(1)} \frac{\omega_{2}}{\dot{\theta}}\right)\left(d_{21}^{(1)}+g_{21}^{(1)} \frac{\omega_{1}}{\dot{\theta}}\right)} \\
& <1<\frac{\omega_{1}}{\dot{\theta}}+\frac{\omega_{2}}{\dot{\theta}}+\frac{\varepsilon \dot{\theta}}{2 \sqrt{\omega_{1} \omega_{2}}} \sqrt{\left(d_{12}^{(1)}-g_{12}^{(1)} \frac{\omega_{2}}{\dot{\theta}}\right)\left(d_{21}^{(1)}+g_{21}^{(1)} \frac{\omega_{1}}{\dot{\theta}}\right)} .
\end{aligned}
$$

6.3. CASE C: COMBINATION RESONANCE OF THE DIFFERENCE TYPE

The stability-instability boundaries in the $\dot{\theta}, \varepsilon$ plane emanate from $\dot{\theta}$ near $\omega_{2}-\omega_{1}$. The stability-instability boundaries are defined by

$$
\frac{\omega_{2}}{\dot{\theta}}-\frac{\omega_{1}}{\dot{\theta}} \pm \frac{\varepsilon \dot{\theta}}{2 \sqrt{\omega_{1} \omega_{2}}} \sqrt{-\left(d_{12}^{(1)}-g_{12}^{(1)} \frac{\omega_{2}}{\dot{\theta}}\right)\left(d_{21}^{(1)}+g_{21}^{(1)} \frac{\omega_{1}}{\dot{\theta}}\right)}=1 \text {. }
$$

The region between the zones is unstable if

$$
\begin{aligned}
\frac{\omega_{2}}{\dot{\theta}}- & \frac{\omega_{1}}{\dot{\theta}}-\frac{\varepsilon \dot{\theta}}{2 \sqrt{\omega_{1} \omega_{2}}} \sqrt{-\left(d_{12}^{(1)}-g_{12}^{(1)} \frac{\omega_{2}}{\dot{\theta}}\right)\left(d_{21}^{(1)}+g_{21}^{(1)} \frac{\omega_{1}}{\dot{\theta}}\right)} \\
& <1<\frac{\omega_{2}}{\dot{\theta}}-\frac{\omega_{1}}{\dot{\theta}}+\frac{\varepsilon \dot{\theta}}{2 \sqrt{\omega_{1} \omega_{2}}} \sqrt{-\left(d_{12}^{(1)}-g_{12}^{(1)} \frac{\omega_{2}}{\dot{\theta}}\right)\left(d_{21}^{(1)}+g_{21}^{(1)} \frac{\omega_{1}}{\dot{\theta}}\right)} .
\end{aligned}
$$

The case A and case B stability-instability zones obtained by Hsu's method are shown in Figures 3 and 4 (as well as additional results), for the physical properties given in section 5. The first zone (Case A) intersects the $\dot{\theta}$ axis at $\dot{\theta}=2 \omega_{1}=1716.6 \mathrm{rad} / \mathrm{s}$. The

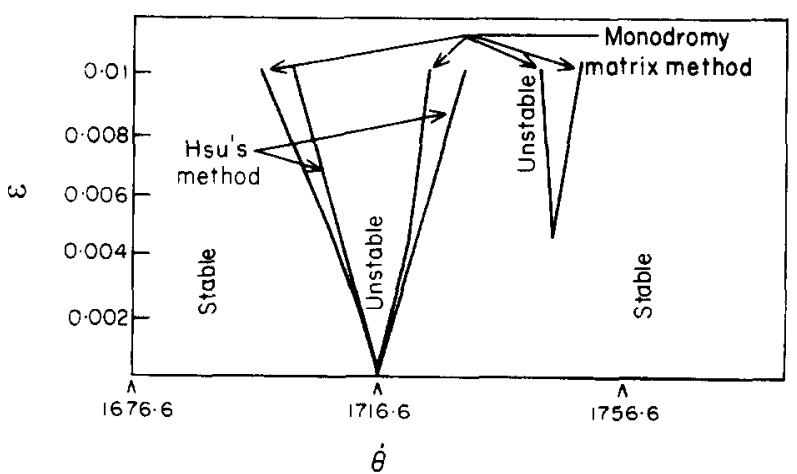

Figure 3. Homogeneous equation instability zones near $\dot{\theta}=2 \omega_{1}$ and $\dot{\theta}=\left(\omega_{1}+\omega_{2}\right) / 2$. 


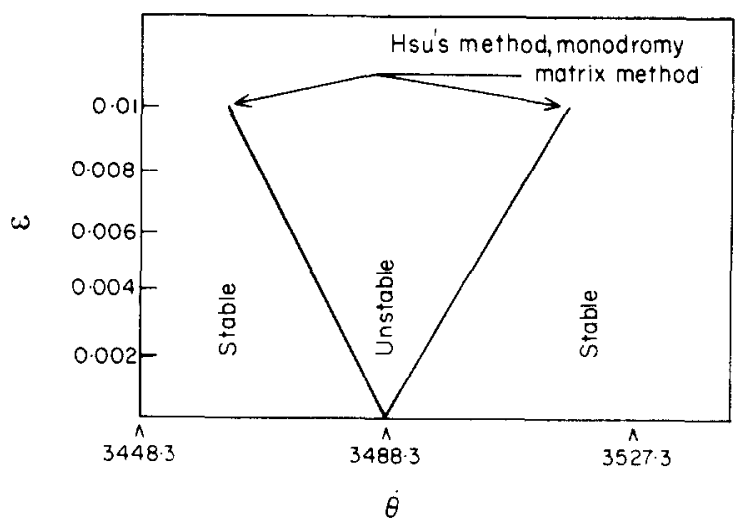

Figure 4. As Figure 3 but near $\dot{\theta}=\omega_{1}+\omega_{2}$.

second zone (Case B) intersects the $\dot{\theta}$ axis at $\dot{\theta}=\omega_{1}+\omega_{2}=3488 \cdot 3 \mathrm{rad} / \mathrm{s}$. No instability zone associated with a crank speed of $\omega_{2}-\omega_{1}$ (Case C) was found. Notice that crank speeds at which the plotted instabilities occur are very high, so high that they are not likely to be encountered in practice.

As shown in reference [18], stability boundaries can be obtained numerically by using the monodromy matrix. An outline of the method follows. It requires the numerical integration over the period of the crank rotation of four (in a two mode approximation) ordinary differential equations for four different sets of initial conditions. This generates a matrix (monodromy matrix) whose eigenvalues must then be determined. The process must be repeated for each $(\dot{\theta}, \varepsilon)$ point and so can be quite computationally intensive. It has the merits of not being restricted to small values of $\varepsilon$, but it is not the most economic approach when analytical results (such as Hsu's) are available. The technique is used here to validate the above results and (as an interesting aside) to obtain some information on the range of validity of Hsu's method. The results are overlayed the perturbation results, and are shown in Figures 3 and 4. Results indicate that Hsu's method is accurate up to about $\varepsilon=0.01$.

It is known (see reference [21]) that for the Mathieu-Hill equation instability zones can also emanate from $\dot{\theta}=2 \omega_{1} / n, n=2,3,4, \ldots$. Such zones, if they exist for the present case, could be of concern in that the instabilities could drop into the range of practical concern. They could be investigated by retaining terms to order $\varepsilon^{n}$ in Hsu's method, but the algebra could be prohibitive. Instead, the authors chose to use the monodromy matrix technique.

The following zones were investigated using $\dot{\theta}$ intervals of $1 \mathrm{rad} / \mathrm{s}$ and $\varepsilon$ intervals of 0.001 .

$\dot{\theta}=2 \omega_{1} / n$. For $n \geqslant 2$ no unstable points were found. This does not mean that such regions do not exist, but that they are so thin as to be all but absent.

$\dot{\theta}=\left(\omega_{1}+\omega_{2}\right) / n, n=2,3,4, \ldots$ For $n=2$ (see Figure 3 ) a zone of instability was found. However, the zone is very thin. The search for $n=3,4$ revealed no further zones of instability.

$\dot{\theta}=\left(\omega_{2}-\omega_{1}\right) / n$. Searches at $n=1$ found no regions of instability (as expected from Hsu's method) and also no unstable region for $n=2,3,4$.

$\dot{\theta}=2 \omega_{2} / n$. For $n=1,2,3$ instability zones were found (corresponding to crank speeds of 5260,2630 and $1753 \mathrm{rad} / \mathrm{s}$ ). No instability regions were observed for $n \geqslant 4$.

The above results show that all zones of instability become so thin as to be invisible for $n \geqslant 4$. It can then be concluded that succeeding regions beyond the instability region 
of the first approximation have progressively less importance for the small crank problem. Since zones where parametric resonance actually occur are beyond normal operating speeds in practical applications, it can be concluded that parametric resonance is not of practical importance when the crank is small. Damping, not included in the analysis but present in every physical system, would further strengthen this conclusion.

One other side result came out of this portion of the study. Many classical techniques (such as the Fourier series method for obtaining Hill's infinite determinant [21]) for determining stability boundaries hinge on utilizing the periodicity of solutions on the boundary. Results from the monodromy matrix search procedure showed that on the boundary for the combination resonance $\omega_{1}+\omega_{2}$, the period varies, so many classical techniques will fail.

\section{NON-HOMOGENEOUS EQUATION STABILITY}

Stability of coupled non-homogeneous equations with periodic coefficients has not been, to the authors' knowledge, fully treated in the existing literature on flexible mechanisms. Gürgöze [12], for example, ignored it in the sewing machine mechanism. Jandratis and Lowen [3] attacked nonhomogeneous equation stability in a four bar biological shaker, but uncoupled the ordinary differential equations by ignoring the coupling terms. They could then apply a Fourier series expansion technique, developed by Kotowski [23], for a forced Mathieu-Hill equation. This study revealed amplification of the solution at speeds $\omega_{1} / n$, where $n$ is a positive integer. This conclusion was also experimentally verified.

Here the coupling terms are not ignored. Hsu's method is used in the Appendix to cover the non-homogeneous case. At $\dot{\theta}=\omega_{1}$, the solution is described by equations (A3), (A7), (A16) and (A17). It is shown therein, within the framework of the first approximation, instabilities (of a different type than in the previous section, in that linear growth is predicted) occur at $\dot{\theta}$ equal to $\omega_{1}$ and $\omega_{2}$. To see whether in fact the forced response is unstable at $\omega_{1}$, the inhomogeneous equations (19) were numerically integrated at $\omega_{1}$, for initial conditions $f_{i}(0)=0, \mathrm{~d} f_{i}(0) / \mathrm{d} \theta=0, i=1,2, \varepsilon=0.01$.

The results for $f_{1}$ shown in Figure 5 verifies that the inhomogeneous equation instability does indeed exist, and that the growth is linear as predicted. Several other cases were also investigated numerically: namely, $\dot{\theta}=\omega_{1} / 2, \omega_{1} / 3, \omega_{1} / 5$. As shown in Figures 6,7

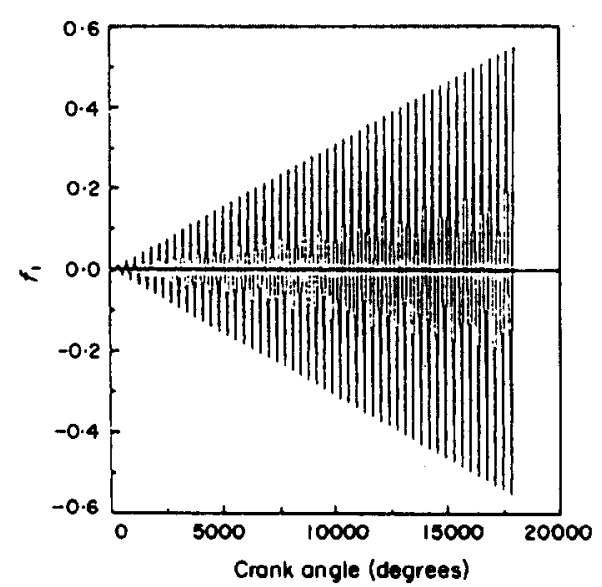

Figure 5. Non-homogeneous equation response at $\dot{\theta}=\omega_{1}, \varepsilon=0.01$. 


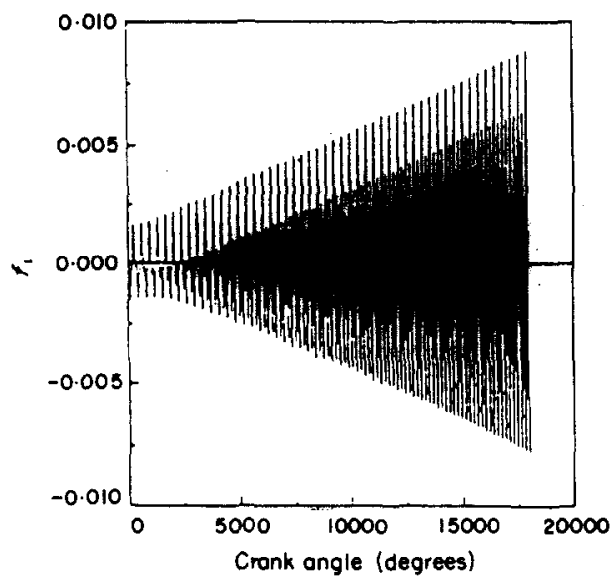

Figure 6. As Figure 5 but at $\dot{\theta}=\omega_{1} / 2$.

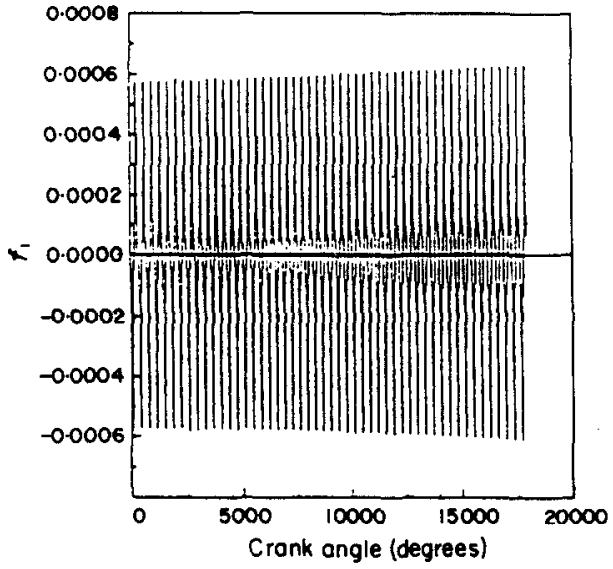

Figure 7. As Figure 5 but at $\dot{\theta}=\omega_{1} / 3$.

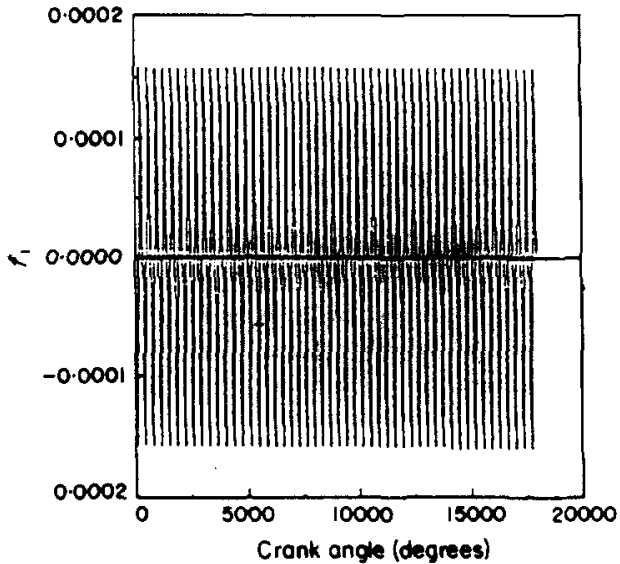

Figure 8. As Figure 5 but at $\dot{\theta}=\omega_{1} / 5$. 
and 8 , respectively, the response also varies linearly with $\theta$. These instabilities were not predicted by Hsu's first order method applied to the nonhomogeneous case, and it is conjectured that they would arise in a higher order expansion. These results also show that the severity of the instability decays as $n$ increases. At $n=5$, the instability is all but absent. The crank speed at $n=5$ is still beyond practical operating speeds.

Actually the parameters set forth in section 5 represent a somewhat flexible configuration. In practice the natural frequencies would be higher than those in the current example, which reinforces the overall conclusion that, at least for small cranks, all instabilities lie outside practical operating speeds.

\section{REFERENCES}

1. M. BADlANi and W. KLEINHENZ 1979 Journal of Mechanical Design 101, 149-153. Dynamic stability of elastic mechanisms.

2. S. C. CHU and K. D. PAN 1975 Journal of Engineering for Industry 97, 542-550. Dynamic response of a high speed slider-crank with an elastic connecting rod.

3. W. G. JANDRATIS and G. G. LOWEN 1979 Journal of Mechanical Design 101, 77-88. The elastic-dynamic behavior of a counterweighted rocker link with an overhanging endmass in a four-bar linkage, Part 1: Theory.

4. B. V. VISCOMI and R. S. AYRES 1971 Journal of Engineering for Industry 93, 251-262. Nonlinear dynamic response of elastic slider-crank mechanism.

5. Z. G. ZHU and Y. CHEN 1983 Journal of Mechanisms, Transmissions, and Automation in Design $105,637-640$. The stability of motion of a connecting rod.

6. W. L. Cleghorn, B. TABARRoK and R. G. Fenton 1984 Mechanism and Machine Theory 19, 307-314. Critical running speeds and stability of high speed flexible mechanisms.

7. W. L. Cleghorn, R. G. FENTON and B. TABARRoK 1984 Mechanism and Machine Theory 19, 417-423. Steady-state vibrational response of high speed flexible mechanisms.

8. C. W. HAM, E. J. CRANE and W. L. ROGERS 1958 Mechanics of Machinery. New York: McGraw-Hill.

9. G. W. SANDOR and A. G. ERDMAN 1984 Advanced Mechanism Design: Analysis and Synthesis, Volume 2 Englewood Cliff, N.J.: Prentice-Hall.

10. S. N. DwIVEDI 1984 Mechanism and Machine Theory 19, 51-59. Application of a Whitworth quick return mechanism for high velocity impacting press.

11. K. W. BUfFINGTON and T. R. KANE, 1985 International Journal of Solids and Structures 21, 617-643. Dynamics of a beam moving over supports.

12. M. GÜRGÖZE 1985 ZAMM 65, 451-454. Ein Beitrag zum Stabilitatsverhalten einer Pendelnden Kurbelschliefe.

13. Y. C. PAN 1988 Ph.D. Dissertation, University of Michigan. Dynamic simulation of flexible robots with prismatic joints.

14. B. M. BAhGAT and K. D. WillmerT 1976 Mechanism and Machine Theory 11, 47-71. Finite element vibrational analysis of planar mechanisms.

15. J. O. SONG and E. J. HAUG 1980 Computer Methods in Applied Mechanics and Engineering 24, 359-381. Dynamic analysis of planar flexible mechanisms.

16. W. J. YOO and J. HAUG 1986 Journal of Mechanisms, Transmissions and Automation in Design 108, 315-322. Dynamics of flexible mechanical systems using vibration and static correction modes.

17. C. S. Hsu 1963 Journal of Applied Mechanics 30, 367-372. On parametric excitation of a dynamical system having multiple degrees of freedom.

18. L. MEIROVITCH 1975 Methods of Analytical Dynamics. New York: McGraw-Hill.

19. A. R. FORSYTH 1927 Calculus of Variation. New York: Dover.

20. D. G. BEALE 1988 Ph.D. Dissertation, University of Michigan. A study of the motion of a flexible rod in a quick return mechanism.

21. V. V. Bolotin 1964 The Dynamic Stability of Elastic Systems. San Francisco: Holden-Day.

22. J. J. STOKER 1950 Nonlinear Vibrations in Mechanical and Electrical Systems. New York: Interscience.

23. G. KoTOWSKI 1943 ZAMM 23, 213-229. Losungen der Inhomogenen Mathieuschen Differentialgleichung mit Periodischer Storfunktion Beliebiger Frequenz. 


\section{APPENDIX: HSU'S METHOD FOR NON-HOMOGENEOUS EQUATIONS}

Hsu's method, as presented in reference [17], was applied only to homogeneous differential equations with periodic coefficients. However, the method can be used to investigate the stability of nonhomogeneous equations with periodic coefficients. Equations (19), with $N=2$, are rewritten in first order form:

$$
\begin{gathered}
\mathrm{d} f_{k} / \mathrm{d} \theta=F_{k}, \\
\frac{\mathrm{d} F_{k}}{\mathrm{~d} \theta}+\frac{\omega_{k}^{2}}{\dot{\theta}^{2}} f_{k}=-\varepsilon\left(\cos \theta \sum_{n=1}^{2} d_{k n}^{(1)} f_{n}+\sin \theta \sum_{n=1}^{2} g_{k n}^{(1)} F_{n}-\frac{\bar{g}_{k 1}}{\Delta_{k k 0}} \sin \theta\right), \quad k=1,2 .
\end{gathered}
$$

The proposed solution is

$$
f_{k}=A_{k}(\theta) \cos \frac{\omega_{k}}{\dot{\theta}} \theta+B_{k}(\theta) \sin \frac{\omega_{k}}{\dot{\theta}} \theta+\varepsilon f_{k}^{(1)}(\theta), \quad k=1,2
$$

where $A_{k}, B_{k}$ and $f_{k}^{(1)}$ are to be determined. There is freedom to impose the following conditions:

$$
\frac{\mathrm{d} A_{k}}{\mathrm{~d} \theta} \cos \frac{\omega_{k}}{\dot{\theta}} \theta+\frac{\mathrm{d} B_{k}}{\mathrm{~d} \theta} \sin \frac{\omega_{k}}{\dot{\theta}} \theta=0
$$

Using this equation, together with equations (A1) and (A3), one obtains

$$
F_{k}=\frac{\omega_{k}}{\dot{\theta}}\left(-A_{k}(\theta) \sin \frac{\omega_{k}}{\dot{\theta}} \theta+B_{k}(\theta) \cos \frac{\omega_{k}}{\dot{\theta}} \theta\right)+\varepsilon \frac{\mathrm{d} f_{k}^{(1)}(\theta)}{\mathrm{d} \theta}
$$

Substituting this into equation (A2) and applying some trigonometric identities yields

$$
\begin{aligned}
&-\frac{\omega_{k}}{\dot{\theta}} \frac{\mathrm{d} A_{k}}{\mathrm{~d} \theta} \sin \frac{\omega_{k}}{\dot{\theta}} \theta+\frac{\omega_{k}}{\dot{\theta}} \frac{\mathrm{d} B_{k}}{\mathrm{~d} \theta} \cos \frac{\omega_{k}}{\dot{\theta}} \theta+\varepsilon\left(\frac{\mathrm{d}^{2} f_{k}^{(1)}}{\mathrm{d} \theta^{2}}+\frac{\omega_{k}^{2}}{\dot{\theta}^{2}} f_{k}^{(1)}\right) \\
&=\varepsilon \frac{\bar{g}_{k 1}}{\Delta_{k k 0}} \sin \theta-\frac{\varepsilon}{2} \sum_{n=1}^{2} d_{k n}^{(1)}\left[A_{n}\left\{\cos \left(\frac{\omega_{n}}{\dot{\theta}} \theta+\theta\right)+\cos \left(\frac{\omega_{n}}{\dot{\theta}} \theta-\theta\right)\right\}\right. \\
&+\left.B_{n}\left\{\sin \left(\frac{\omega_{n}}{\dot{\theta}} \theta+\theta\right)+\sin \left(\frac{\omega_{n}}{\dot{\theta}} \theta-\theta\right)\right\}\right] \\
&-\frac{\varepsilon}{2} \sum_{n=1}^{2} g_{k n}^{(1)} \frac{\omega_{n}}{\dot{\theta}}\left[A_{n}\left\{\cos \left(\frac{\omega_{n}}{\dot{\theta}} \theta+\theta\right)-\cos \left(\frac{\omega_{n}}{\dot{\theta}} \theta-\theta\right)\right\}\right. \\
&\left.+B_{n}\left\{\sin \left(\frac{\omega_{n}}{\dot{\theta}} \theta+\theta\right)-\sin \left(\frac{\omega_{n}}{\dot{\theta}} \theta-\theta\right)\right\}\right] .
\end{aligned}
$$

Equations (A4) and (A6) completely define a small parameter first order approximation of equations (19), but transformed to variables $A_{k}, B_{k}$ and $f_{k}^{(1)}$. In accordance with Hsu's method, solutions are obtained by solving the system equations that are coefficients of $\varepsilon^{0}$ and $\varepsilon^{1}$. In general, these solutions are correct, except when $\dot{\theta}$ is near crank speeds related to the first and second natural frequency. See section 6 for the homogeneous equation stability results.

Inhomogeneous equation instability can be studied from the "perturbational equations", which are obtained from the coefficients of $\varepsilon^{1}$ of equation (A6). The 
homogeneous solutions of these equations are

$$
f_{1}^{(1)}=C_{1} \cos \frac{\omega_{1}}{\dot{\theta}} \theta+C_{2} \sin \frac{\omega_{1}}{\dot{\theta}} \theta, \quad f_{2}^{(1)}=C_{3} \cos \frac{\omega_{2}}{\dot{\theta}} \theta+C_{4} \sin \frac{\omega_{2}}{\dot{\theta}} \theta,
$$

where $C_{1}, C_{2}, C_{3}$ and $C_{4}$ are constants. The particular integrals of the perturbational equations are

$$
\begin{aligned}
f_{1}^{(1)}= & -\frac{1}{2} \sum_{n=1}^{2}\left[\frac{\dot{\theta}^{2}}{\omega_{1}^{2}-\left(\omega_{n}+\dot{\theta}\right)^{2}}\left\{T_{1 n} \cos \left(\frac{\omega_{n}}{\dot{\theta}} \theta+\theta\right)+V_{1 n} \sin \left(\frac{\omega_{n}}{\dot{\theta}} \theta+\theta\right)\right\}\right. \\
& \left.+\frac{\dot{\theta}^{2}}{\omega_{1}^{2}-\left(\omega_{n}-\dot{\theta}\right)^{2}}\left\{U_{1 n} \cos \left(\frac{\omega_{n}}{\dot{\theta}} \theta-\theta\right)+W_{1 n} \sin \left(\frac{\omega_{n}}{\dot{\theta}} \theta-\theta\right)\right\}\right] \\
& +\frac{\dot{\theta}^{2}}{\omega_{1}^{2}-\dot{\theta}^{2}} \frac{\bar{g}_{11}}{\Delta_{110}} \sin \theta, \\
f_{2}^{(1)}= & -\frac{1}{2} \sum_{n=1}^{2}\left[\frac{\dot{\theta}^{2}}{\omega_{2}^{2}-\left(\omega_{n}+\dot{\theta}\right)^{2}}\left\{T_{2 n} \cos \left(\frac{\omega_{n}}{\dot{\theta}} \theta+\theta\right)+V_{2 n} \sin \left(\frac{\omega_{n}}{\dot{\theta}} \theta+\theta\right)\right\}\right. \\
& \left.+\frac{\dot{\theta}^{2}}{\omega_{2}^{2}-\left(\omega_{n}-\dot{\theta}\right)^{2}}\left\{U_{2 n} \cos \left(\frac{\omega_{n}}{\dot{\theta}} \theta-\theta\right)+W_{2 n} \sin \left(\frac{\omega_{n}}{\dot{\theta}} \theta-\theta\right)\right\}\right] \\
& +\frac{\dot{\theta}^{2}}{\omega_{2}^{2}-\dot{\theta}^{2}} \frac{\bar{g}_{21}}{\Delta_{220}} \sin \theta,
\end{aligned}
$$

where

$$
\begin{array}{ll}
T_{k n}=\left(d_{k n}^{(1)} A_{n}+g_{k n}^{(1)} \frac{\omega_{n}}{\dot{\theta}} A_{n}\right), & U_{k n}=\left(d_{k n}^{(1)} A_{n}-g_{k n}^{(1)} \frac{\omega_{n}}{\dot{\theta}} A_{n}\right), \\
V_{k n}=\left(d_{k n}^{(1)} B_{n}+g_{k n}^{(1)} \frac{\omega_{n}}{\dot{\theta}} B_{n}\right), & W_{k n}=\left(d_{k n}^{(1)} B_{n}-g_{k n}^{(1)} \frac{\omega_{n}}{\dot{\theta}} B_{n}\right) .
\end{array}
$$

Consider the situation when $\omega_{1}^{2}-\theta^{2}$ is nearly equal to zero. A solution is sought for $\omega_{1}$ near $\dot{\theta}$, as described by $\omega_{1}+\varepsilon \gamma \dot{\theta}=\dot{\theta}$, where $\gamma$ is a finite real number. In Hsu's prodecure, the "offending" term in equation (A8) is removed from the perturbational equations, and associated with the "variational equations" (the equations obtained from the coefficients of $\varepsilon^{0}$ in equation (A6)). The resulting equations are

$$
\begin{gathered}
\frac{\mathrm{d} A_{1}}{\mathrm{~d} \theta} \cos \frac{\omega_{1}}{\dot{\theta}} \theta+\frac{\mathrm{d} B_{1}}{\mathrm{~d} \theta} \sin \frac{\omega_{1}}{\dot{\theta}} \theta=0, \quad \frac{\mathrm{d} A_{2}}{\mathrm{~d} \theta} \cos \frac{\omega_{2}}{\dot{\theta}} \theta+\frac{\mathrm{d} B_{2}}{\mathrm{~d} \theta} \sin \frac{\omega_{2}}{\dot{\theta}} \theta=0 \\
-\frac{\omega_{1}}{\dot{\theta}} \frac{\mathrm{d} A_{1}}{\mathrm{~d} \theta} \sin \frac{\omega_{1}}{\dot{\theta}} \theta+\frac{\omega_{1}}{\dot{\theta}} \frac{\mathrm{d} B_{1}}{\mathrm{~d} \theta} \cos \frac{\omega_{1}}{\dot{\theta}} \theta=\varepsilon \frac{\bar{g}_{11}}{\Delta_{110}} \sin \theta \\
-\frac{\omega_{2}}{\dot{\theta}} \frac{\mathrm{d} A_{2}}{\mathrm{~d} \theta} \sin \frac{\omega_{2}}{\dot{\theta}} \theta+\frac{\omega_{2}}{\dot{\theta}} \frac{\mathrm{d} B_{2}}{\mathrm{~d} \theta} \cos \frac{\omega_{2}}{\dot{\theta}} \theta=0 .
\end{gathered}
$$

Solving these equations gives, for $\gamma=0$ (i.e., $\omega_{1}=\dot{\theta}$ ),

$$
A_{1}=\varepsilon \frac{\bar{g}_{11} \dot{\theta}}{2 \omega_{1} \Delta_{110}}\left(\theta-\frac{\sin 2 \theta}{2}\right)+C_{5}, \quad B_{1}=\varepsilon \frac{\bar{g}_{11} \dot{\theta}}{4 \omega_{1} \Delta_{110}} \cos 2 \theta+C_{6},
$$

where $C_{5}$ and $C_{6}$ are constant. Note that equation (A16) predicts an unstable response in that $A_{1}$ grows linearly with time $(\theta)$. Inspection of equation (A9) shows that a similar instability occurs at $\dot{\theta}=\omega_{2}$. 
If $\gamma \neq 0$, one obtains the bounded solutions:

$$
\begin{aligned}
& A_{1}=\varepsilon \frac{\bar{g}_{11} \dot{\theta}}{2 \omega_{1} \Delta_{110}}\left\{\frac{\sin \varepsilon \gamma \theta}{\varepsilon \gamma}-\frac{\sin (2 \theta-\varepsilon \gamma \theta)}{2-\varepsilon \gamma}\right\}+C_{5}, \\
& B_{1}=-\varepsilon \frac{\bar{g}_{11} \dot{\theta}}{2 \omega_{1} \Delta_{110}}\left\{-\frac{\cos (2 \theta-\varepsilon \gamma \theta)}{2-\varepsilon \gamma}-\frac{\cos \varepsilon \gamma \theta}{\varepsilon \gamma}\right\}+C_{6} .
\end{aligned}
$$

